\title{
Learning and transfer in matching-to-sample tasks
}

\author{
TAKESHI SUGIMURA ${ }^{1}$ \\ Deparlment of Psychology, Nara Unizersity of Education, Nara 630
}

\begin{abstract}
One hundred and twenty-eight kindergarten children were trained on an identity or an oddity task with color and form dimensions to $6 / 6$ or $6 / 6+30$ correct responses and then tested on the transfer task with the same rule-different dimension (S-D task) or the task with the different rule-same dimension (D-S task). When the subjects were trained to $6 / 6$ correct responses and on the oddity rule, the D-S transfer task was learned faster than the S-D task. When the subjects were trained to $6 / 6+30$ correct responses and on the identity rule, the two tasks were learned almost at the same rate. The findings were explained by taking account of the strength of rule and dimensional responses learned in the original task and of the property of the rule learned, and were discussed in relation to the rule and the attention models of relational learning.
\end{abstract}

Key words: rule (identity and oddity) learning, dimensional response, transfer, rule model, attention model.

In an extensive review of literature on identity-difference discrimination learning, House, Brown, and Scott (1974) proposed a chaining attentional model. According to this model, oddity learning is repre. sented as acquisition on a chain of three components: (a) attention to a relevant vehicle dimension which carries the oddity relation, (b) attention to the oddity relation among the cues on the relevant dimension, and (c) the instrumental response to the odd stimulus. An important assumption in this model is that for the solution of oddity tasks attention to the oddity relation must be preceded by atten. tion to the relevant dimension. They suggested that this model could be applicable to other types of identity-difference learning with only minor variations.

In an earlier study Brown (1970) found that second grade children who were overtrained on an original task learned very rapidly both an intradimensional and an extradimensional oddity tasks. On the basis of this finding Brown suggested the

1 The author is indebted to Miss Taemi Kouchi for collecting and processing the data and to Mrs. Yoko Sato for helpful comments on an early draft of this paper. possibility of reversal in the sequential order of the two attentional (or observing) responses: "Once the relational observing response (suggested by House) reached asymptote, the sequential order of the two observing responses would be reversed and the problem would become one of the searching for the oddity relationship (e.g., "find the one that's odd"), followed by a search for the dimension carrying the odd cue (p. 317)." Recently Bowers (1976) proposed a model which is referred to as a rule model by generalizing the Brown's suggestion. In this model it is assumed that subjects first select a general problem-solving rule, then attend to a vehicle dimension, and finally choose a cue. Although different terminologies (rule and relation) are used, the two components assumed in the rule and the attention models have very similar implications. However, there is an important difference in the order that the two components are processed. The rule model assumes that subjects select a rule first and then attend to a vehicle dimension, whereas the attention model assumes that subjects attend to a vehicle dimension and then attend to a relation. An assumption common to both models is that processing 
the second components is conditional on that of the first components.

In an attempt to compare the two models, Bowers (1976) deviced four transfer tasks according to whether the rule (oddity vs. discrimination) or the dimension (color vs. form) was the same or different across the original and the transfer tasks, and considered the number of steps (components) which subjects had to change from the original task to learn the transfer task. If both the rule and the dimension are not changed on the transfer task, the number of steps changed is zero for both the rule and the attention models. If both the rule and the dimension are changed, subjects must change the two steps (rule and dimension) for the two models. Thus one can not differentiate the two models under these transfer conditions. If the dimension is changed but the rule is not, on the other hand, subjects must change one step (dimension) for the rule model and two steps (dimension and rule) for the attention model. For the attention model, subjects must resample the relation (rule) whenever they change the dimension since attention to the relations is conditional on that to the relevant dimension. If the rule is changed but the dimension is not, subjects must change one step (rule) for the attention model and two steps for the rule model. For the rule model, subjects must resample the dimension whenever they change the rule since attention to the dimension is conditional on the rule selection. As learning rate of the transfer task is assumed to depend on the number of changed steps, the rule model predicts that the transfer task with the same rule and the changed dimension will be learned faster than the task with the changed rule and the same dimension, whereas the attention model predicts the reverse result. Bowers (1976) obtained the result supporting the rule model and emphasized the importance of studying rules in children's discrimination learning.
The present study was designed to examine whether Bowers' finding could be also obtained when different subjects and tasks were used. As regards the subjects fifth and sixth graders were used in Bowers' study while kindergarten children were used in this study. As regards the tasks the subjects in Bowers' study were transferred from a three-position oddity task to a three-choice discrimination or vice versa while the subjects in this study were transferred from an identity task to an oddity or vice versa. As mentioned earlier, the changed rule-same dimension and the same rule-changed dimension transfer conditions are decisive for comparing the attention with the rule model. So, these two transfer conditions were provided in the present study. In addition, effects of overtraining on transfer tasks were examined after the Brown's suggestion (1970) that subjects first search a rule and then attend to the dimension after overtraining.

\section{Method}

\section{Design and Subjects}

A $2 \times 2 \times 2 \times 2$ factorial design was used, which incorporated the rule in original task (identity vs. oddity), the relevant dimension in original task (color vs. form), the degree of training in original task (6/6 vs. $6 / 6+30$ correct), and the type of transfer task (same-different vs. differentsame). The subjects were 128 (64 males and 64 females) kindergarten children whose mean age was $6: 3(5: 8-6: 7)$. They were assigned to 16 subgroups of four males and four females approximately matching the mean ages. Additional seven children were discarded from the experiment for failure to reach the criterion in the original learning.

\section{Materials}

Each set had three stimuli which were put on a $10 \times 13-\mathrm{cm}$ white card in a triangular array. A sample stimulus was put on the top of the card and two choice 
stimuli were put on the bottom of the card in a horizontal array. Two tasks with color and form dimensions were provided, in each of which the following four response rules were available for learning the tasks: color-identity, form-identity, color-oddity, and form-oddity rules.

Task A was constructed by combining an orange square $(3.0 \mathrm{~cm}$ in side), an orange $T(3.4 \mathrm{~cm}$ in height and $1.0 \mathrm{~cm}$ in width), a green square, and a green $T$. An orange $T$ and a green square were used as the samples four times, respectively. When an orange $T$ was used as the sample, a green $T$ and an orange square or an orange $T$ and a green square were used as the choice stimuli. Four sets were constructed by exchanging the left-right positions of the choice stimuli. For the sets with a green $T$ and an orange square as the chioce stimuli the selection of a green $T$ was regarded as an identity response on the form dimension or an oddity response on the color dimension. The selection of an orange square was regarded as an identity response on the color dimension or an oddity response on the form dimension. For the sets with an orange $\mathrm{T}$ and a green square as the choice stimuli, the selection of an orange $T$ was regarded as an identity response on both the color and the form dimensions, and the selection of a green square was regarded as an oddity response on both dimensions. When a green square was used as the sample, an orange square and a green $\mathrm{T}$ or a green square and an orange $\mathrm{T}$ were used as the choice stimuli. Four sets were constructed by exchanging the left-right positions of the choice stimuli. For the sets with an orange square and a green $\mathrm{T}$ as the choice stimuli, the selection of an orange square was regarded as an identity response on the form dimension or an oddity response on the color dimension. The selection of a green $T$ was regarded as an identity response on the color dimension or an oddity response on the form dimension. For the sets with an orange $\mathrm{T}$ and a green square as the choice stimuli, the selection of an orange $\mathrm{T}$ was regarded as an oddity response on both the color and the form dimensions, and the selection of a green square was regarded as an identity response on both dimensions. These eight sets were used repeatedly three times for providing a total of 24 sets.

Task $B$ was constructed by combining a blue circle $(3.2 \mathrm{~cm}$ in diameter $)$, a blue $\mathrm{X}$ (3.4 cm in length and $1.1 \mathrm{~cm}$ in width), a pink circle, and a pink $X$. In the same way as in Task $A$, a total of 24 sets were provided. Half of the sets had a blue $X$ as the sample and the remaining sets had a pink circle. In half of the sets the selection of one choice stimulus was regarded as an identity response on the form dimension or an oddity response on the color dimension, and the selection the other stimulus was regarded as an identity response on the color dimension or an oddity response on the form dimension. In the remaining sets the selection of one choice stimulus was regarded as an identity response on both dimensions and the selection of the other stimulus was regarded as an oddity response on both dimensions.

Twenty-four sets of each task were arranged with the restriction that (a) a particular sample stimulus did not appear successively more than two trials, (b) the correct choice stimuli did not appear on the same position successively more than three trials, (c) a particular choice stimulus did not appear on the same position successively more than two trials, (d) a particular value on one dimension did not appear on the same position successively more than three trials, and (e) the correct stimuli did not appear in the pattern of color, form, and position alternations.

\section{Procedure}

The subjects were tested individually in a small room of their school. The subject was presented the first card of the 
original task with the instructions, "Let's play a card game. (The experimenter points to the two choice stimuli altcrnatively.) One of these pictures is always correct and the other is always incorrect. (The experimenter points to the sumple stimulus.) Look at this picture carefully and point to the lower one that you think correct. If you point to a correct one, I will say 'Hit' but if you point to an incorrect one, I will say 'Miss'. Please try to get as many as 'Hit's as possible." Then the subject was presented the cards one by one at his response rate. When the subject responded incorrectly in the original learning, he was given the instructions for correction, "Miss. Look at this picture carefully. (The experimenter points to the sample and then the correct choice stimulus.) This is correct." All subjects were trained to reach a criterion of six successive correct responses or 48 trials. If the subject did not reach the criterion within 48 trials, he was discarded and replaced by an additional subject. Immediately after reaching the criterion, half of the subjects were given the transfer task to reach a criterion of eight successive correct responses or 48 trials. The remaining subjects were given additional 30 overtraining (correct) trials beyond the criterion and then the transfer task.

In the original learning half of the subjects were trained on Task A to learn either one of the four response rules and the remaining subjects were trained on Task B. The subjects trained on Task $A$ in the original learning were given Task $B$ in the transfer and the subjects trained on Task B were given Task A. For the same-different transfer task the rule (identity or oddity) was the same as the original learning but the relevant dimension (color or form) was changed from the original to the transfer task. For the differentsame transfer task the rule was changed from the original to the transfer task but the relevant dimension was the same as the original task.
Results

\section{Original Learning}

A 2 (rule) $\times 2$ (dimension) analysis of variance for the number of trials to criterion was performed by the use of $\sqrt{ } . Y+0.5$ transformed scores, which showed that the main effects of rule and dimension were significant at $5 \%$ level with $F(1$, $124)=6.42$ and $1 \%$ level with $F(1,124)$ $=8.92$, respectively. The identity rule (mean trial $=3.22$ ) was learned faster than the oddity rule (mean trial $=4.59$ ), and the form dimension (mean trial=3.08) was learned faster than the color dimension (mean trial $=4.73$ ). To check whether the overtrained subjects overlearned reliably the original task, the number of errors during the overtraining trials was counted. The mean number of errors was 0.50 (color-identity), 1.19 (coloroddity), 0.56 (form-identity), and 0.88 (form-oddity) for a total mean of 0.8 . These results showed that the overtrained subjects responded correctly far more times than the criterion subjects.

\section{Transfer}

A $2 \times 2 \times 2 \times 2$ analysis of variance was performed for $\sqrt{X+0.5}$ transformed scores of trials to criterion. The main effect of transfer type was significant at $5 \%$ level with $F(1,112)=4.09$, which showed that the different-same task (mean trial=12.32) was learned faster than the same-different task (mean trial $=19.16$ ). Three firstorder interactions were also significant; $F(1,112)=5.26, p<.05$ for the original rule by degree of training, $F(1,112)=$ $7.64, p<.01$ for the original rule by transfer type, and $F(1,112)=4.33, p<.05$ for the degree of training by transfer type, respectively. Among these interactions the present experiment is especially concerned with the second and the third interactions, and these are shown in Table 1. Several $t$ tests were performed to examine the simple effects by the use of the error term (3.39). The interaction of degree of 
Table 1

Mean numbers of trials to criterion in the transfer tasks

\begin{tabular}{|c|c|c|c|c|c|}
\hline \multicolumn{2}{|c|}{ 'Transfer condition } & \multicolumn{4}{|c|}{ Original learning } \\
\hline Rule & Dimension & Criterion & Overtraining & Identity & Oddity \\
\hline Same & Diflerent & 21.9 & 16.4 & 14.1 & 24.2 \\
\hline Dillerent & Salne & 10.0 & 14.7 & 15.2 & 9.4 \\
\hline
\end{tabular}

Notes: Same-Different in the transfer condition denotes that the rule in the transfer task was the same as the original task (identity $\rightarrow$ identity or oddity $\rightarrow$ oddity) but the relevant dimension was changed from the original to the transfer task (color $\rightarrow$ form or form $\rightarrow$ color). Different-Same denotes that the rule was changed from the original to the transfer task (identity $\rightarrow$ oddity or oddity $\rightarrow$ identity) but the relevant dimension was the same as the original task. Criterion and Overtraining in the original learning denote that the subjects were trained on the original task to six successive correct responses $(6 / 6)$ and $6 / 6$ plus 30 correct responses, respectively.

training and transfer type showed that for the criterion subjects the different-same task was learned significantly faster than the same-different task $(t=2.89, d f=112$, $p<.01)$ while the two tasks were learned at almost the same rate for the overtrained subjects. The interaction of original rule and transfer type showed that for the original oddity-rule the different-same task was learned significantly faster than the same-different task $(l=3.39, d f=112, p<$ $.01)$ while the two tasks were learned at almost the same rate for the identity rule. The same-different task was learned significantly faster for the original identityrule than for the oddity rule $(t=2.65$, $d f=112, p<.01$ ).

\section{Discussion}

The finding that the different-same task was learned faster than the same-different task seems to be consistent with the attention model (House et al., 1974) rather than the rule model (Bowers, 1976). According to the attention model, the number of steps to be changed from the original task to learn the transfer task is one for the different-same task and two for the same-different task. So the former task is learned faster than the latter one. However, an alternative explanation which takes account of the transfer effects of the original rule and dimension may be more plausible. On the tasks used in the present experiment transfer effects of the original dimension as well as the original rule are expected since the relevant dimension in the original task becomes irrelevant and the irrelevant dimension becomes relevant. The present result is interpreted as showing that the original dimension had stronger negative effect on the transfer learning than the original rule.

Table 1 shows that this negative effect of the original dimension was strong when the subjects were trained to reach the $6 / 6$ criterion. This finding is explained to show that the change of the relevant dimension interfered with the transfer of the original rule since the rule was not well learned at this stage of training. The result that the same-different task became easier (though not significant) after overtraining may be attributed to the fact that the negative effect of the original dimension was weakened because the original rule was learned well by overtraining. As mentioned earlier, Brown (1970) suggested that subjects first search a rule and then attend to the dimension after overtraining. If so, it is predicted that the same-different task is learned faster than the different-same task after overtraining. As the two tasks were learned at almost the same rate after over- 
training, Brown's suggestion was not substantiated. If the subjects will be more overtrained than this experiment, the predicted result may be obtained.

Table 1 also shows that the negative effect of the original dimension was stronger when the subjects were trained on the oddity rule in the original task than when they were trained on the identity rule. In particular, it is of great interest to mention that even when the subjects were transferred within the same (identity or oddity) rule, the identity-identity transfer task was learned singificantly faster than the oddity-oddity transfer task. As the subjects have to maintain the original rule and apply it to the new dimension in learning these transfer tasks, the obtaincd result shows that the maintenance and application of the original rule were more difficult for the oddity rule than for the identity rule. Two possibilities may be suggested to explain this result: (a) even when the subjects were given the tasks at a certain level of training, the oddity rule was learned more weakly than the identity rule, and (b) the oddity rule was learned on the basis of perceptual (or dimension-specific) rather than conceptual relations. It may be suggested, in any case, that the two rules are of different nature in the learning processes. When the original task was trained on the oddity rule, the different-same task was learned faster than the same-different task, which showed that the changed dimension had greater negative effects than the changed rule. The transfer from the oddity to the identity rule within the same dimension was easier (though not significant) than that from the identity to the oddity rule. This may be attributed to the fact that the subjects preferred the identity to the oddity rule since the identity rule was learned faster than the oddity rule in the original task.

Although Bowers (1976) obtained the result that the same-different task was learned faster than the different-same task when fifth and sixth graders were transferred from the oddity to the discrimination rule or vice versa in the two-dimensional (color and form) tasks, the present study showed the reverse result when kindergarten children were transferred from the oddity to the identity rule or vice versa in the two-dimensional tasks. The obtained discrepancy may be explained in the following ways. (1) As regards the subject variable, kindergartners were more subject to the dimensional control than graders. In other words, kindergartners learned a dimension-specific (or perceptual) rule whereas graders could learn a general (or conceptual) rule. Thus the transfer with the changed dimension was easier for Bowers' study but was more difficult for this experiment. (2) As regards the rule variable, the degree of change of the rules was greater for Bowers' than for this experiment. That is, Bowers' subjects were transferred to the rules with different properties whereas the present subjects were transferred to the opposite rules within the same property. In relation to this, Brown and Scott (1972) found that the transfer from identity to oddity was easier than that from identity to small. Thus the transfer with the changed rule was easier for the present study but was more difficult for Bowers' study.

Although the present study used the transfer paradigm proposed by Bowers (1976), this paradigm seems to be inappropriate for testing the rule model since the transfer performances obtained in this study varied depending on several other factors rather than the processing order of the rule and the dimension. The present findings can be explained by taking account of the strength of rule and dimensional responses learncd in the original task and the property of the rule learned. It is important to note that oddity and identity tasks can eventually be solved by acquiring the rule but not by attending to the dimension. Therefore, the effects of stimulus dimensions on 
rule learning should be analyzed in further studies. Although both the rule (Bowers, 1976) and the attention (House et al., 1974) models assume that the rule and the dimension are two separable components in the learning process and are processed in the sequential manners, it may be more plausible to assume that both components are not separable and processed in a simultaneous (or parallel) manner.

\section{References}

Bowers, N. P. 1976 Rule learning and transfer in oddity and discrimination problems. Child $D_{\ell-}$ velopment, 47, 1130-1137.

Brown, A. L. 1970 Transfer performance in children's oddity lcarning as a function of dimensional preference, shift paradigm and overtraining. Journal of Experimental Child Psychology, 9, 307-319.

Brown, A. L., \& Scott, M. S. 1972 Transfer between the oddity and relative size concepts: Reversal and extradimensional shifts. Journal of Experimental Child Psychology, 13, 150-367.

House, B. J., Brown, A. L., \& Scott, M. S. 1974 Children's discrimination learning based on identity or difference. In H. W. Reese (Ed.), Advances in child development and behavior. Vol. 9. New York: Academic Press. Pp. 1-45.

(Received Jan. 22, 1982; accepted Jan. 22, 1983) 